\title{
Isolation of Vibrations Transmitted to a LIDAR Sensor Mounted on an Agricultural Vehicle to Improve Obstacle Detection
}

\author{
C.F. Periu ${ }^{1}$, A. Mohsenimanesh ${ }^{2}$, C. Laguë ${ }^{2 *}$ and N.B. McLaughlin ${ }^{3}$ \\ ${ }^{1}$ MacLean Engineering \& Marketing Co. Canada \\ ${ }^{2}$ Department of Mechanical Engineering, Faculty of Engineering, University of Ottawa, Ottawa, ON K1N 6N5 Canada \\ ${ }^{3}$ Eastern Cereal and Oilseed Research Centre, Agriculture and Agri-Food Canada, Ottawa, ON K1A 0C6 Canada \\ *Email: Claude.Lague@uottawa.ca \\ http://dx.doi.org/10.7451/CBE.2013.55.2.33
}

Periu, C.F., A. Mohsenimanesh, C. Laguë and N.B. McLaughlin. 2013. Isolation of Vibrations Transmitted to a LIDAR Sensor Mounted on an Agricultural Vehicle to Improve Obstacle Detection. Canadian Biosystems Engineering/Le génie des biosystèmes au Canada 55: 2.33-2.42. LIDAR (LIght Detection And Ranging) technology can be used on autonomous agricultural vehicles for guidance and obstacle detection purposes. However, the quality of LIDAR measurements can be affected by mechanical vibrations induced by the operation of these vehicles on uneven terrain. The objective of this study was to develop a stabilizing system and to evaluate its effectiveness at reducing the transmission of mechanical vibrations to a LIDAR sensor installed on an agricultural tractor for the purpose of reducing the positioning error of obstacles during field operation. Special support bars (S) and stabilization system (SS) were designed for a SICK LMS 291-S14 LIDAR sensor mounted on an agricultural tractor. The positioning error of the sensor was assessed in field experiments by determining the difference between the known location of obstacles and their corresponding estimated locations from the sensor measurements. Increasing tractor speed had a negative effect on the accuracy of the sensor with an increase in the positioning error of up to $27 \%$. The addition of the S system positively affected the accuracy of the sensor and resulted in a $41 \%$ decrease of the average positioning error from 340 to 201 $\mathrm{mm}$. Finally, the addition of the SS system decreased the average positioning error by $57 \%$ from 382 to $161 \mathrm{~mm}$. Keywords: Laser Measurement System, object detection, vibrations, stabilization system, autonomous, vehicles, safety.

On fait appel à la technologie LIDAR (détection et télémétrie par ondes lumineuses) pour assurer la navigation de véhicules autonomes ainsi que pour détecter de possibles obstacles. Dans le cas d'applications agricoles, les vibrations induites par les machines ainsi que par leur opération en terrain accidenté peuvent affecter l'efficience des systèmes LIDAR de façon négative. Cette étude visait à évaluer l'efficacité d'un système de montage et de stabilisation spécialement conçu pour réduire la transmission de ces vibrations à un capteur LIDAR installé sur un tracteur agricole dans le but de réduire les erreurs de localisation d'obstacles au champ. Des barres de support (S) et un système de stabilisation (SS) spécialisé ont été développés pour un capteur LIDAR SICK LMS 291-S14 utilisé pour la détection de quatre obstacles placés à différents endroits dans des champs agricoles. Un protocole expérimental a été mis en place pour évaluer les erreurs de localisation d'obstacles détectés par le capteur sous différentes conditions de terrain et d'opération. Ces erreurs de localisation ont été déterminée à partir de la différence entre la position exacte des quatre obstacles et leurs positions estimées correspondantes telles que déterminées par le capteur. Les résultats obtenus ont démontré que l'accroissement de la vitesse d'opération du tracteur a résulté en une réduction de la précision $\mathrm{du}$ capteur en raison d'un accroissement de l'erreur de positionnement atteignant $27 \%$. L'ajout du système de support S a permis de réduire la valeur moyenne de l'erreur de positionnement de $41 \%$ (de 340 à $201 \mathrm{~mm}$ ). Enfin, l'utilisation du système de stabilisation SS a résulté en une réduction de l'erreur de positionnement moyenne de 382 à $161 \mathrm{~mm}$ ou 57\%. Motsclés: système de détection au laser, détection d'obstacles, vibrations, système de stabilisation, autonome, véhicules, sécurité.

\section{INTRODUCTION}

Efforts to develop autonomous guidance and operating systems for agricultural vehicles are motivated by the reduced availability of qualified farm workers and by the desire for higher production efficiency and increased safety. While autonomous agricultural vehicles can improve operator safety by removing human operators from the vehicles, their use can also result in other safety concerns related to the risks of collisions with humans, animals, or other vehicles in their vicinity. Therefore, the ability of autonomous agricultural vehicles to detect and identify obstacles and to take appropriate collision avoidance actions is critical. Appropriate obstacle detection for agricultural vehicles relies on machine vision, image processing, and mitigation of adverse operating conditions such as machine vibration.

Machine vision systems are used to measure the relative position and heading of vehicles by means of image sensors. There are several aspects of machine vision based sensing and different types of sensor modalities that can be selected to provide the guidance information. Optimum positioning of the sensor on the vehicle requires an understanding of the geometric relationship between the image sensor, the vehicle and the field-of-view that the sensor uses to provide guidance information ( $\mathrm{Li}$ et al. 2009). Machine vision systems are most commonly used for obstacle detection, mapping, and navigation. The LIDAR technology has been used as a fundamental component of autonomous agricultural vehicles (Doerr 2009; Doerr et al. 2014; Lee and Ehsani 2008; Barawid et al. 2007; Subramanian et al. 2006; Kise et al. 2005; Carmer et al. 1996). 


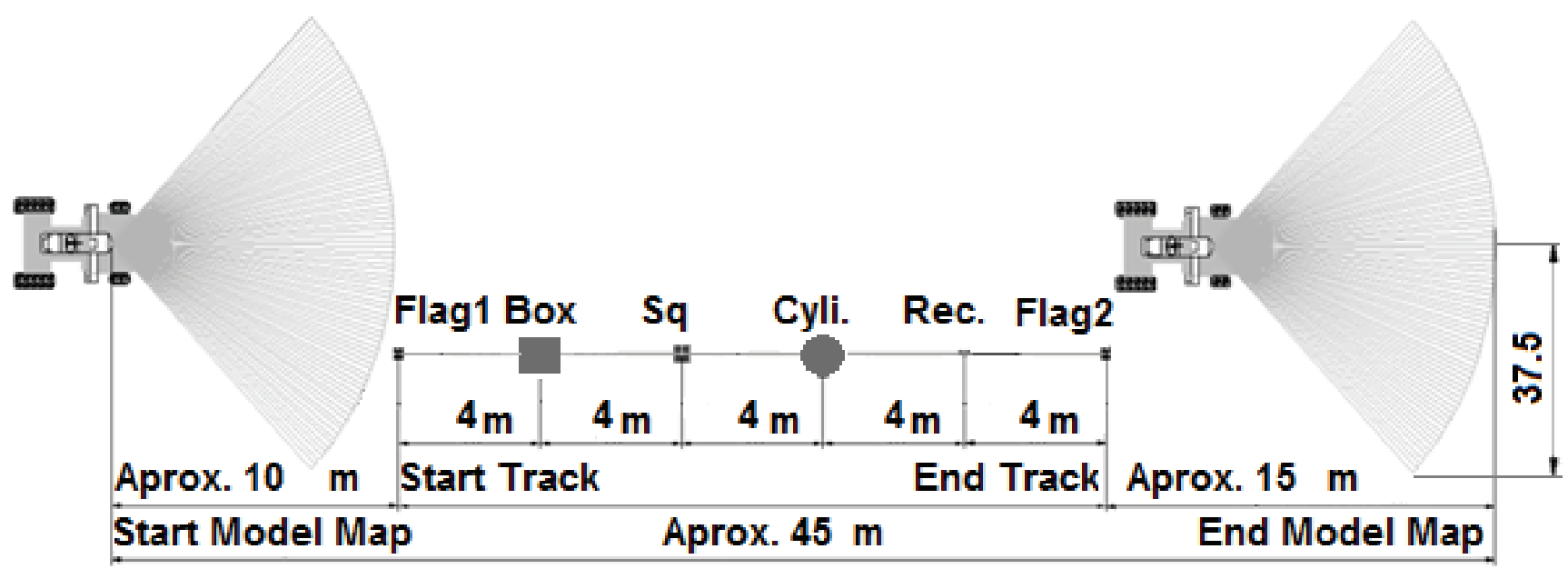

All dimentions are in $\mathbf{m}$

Fig. 1. Top view of the model map created by LIDAR sensor including the position of objects, flags and their dimensions.

Image processing is defined as a set of computational techniques for analyzing, enhancing, compressing, and reconstructing images and has extensive applications in many areas, including robotics and machine vision. Morphological operations are theoretical approaches to image analysis based on shape, and are commonly applied in machine vision and automatic object detection (Burdick 1997). Several methods originating from the mobile robot and computer vision fields have been studied for application in obstacle detection (Barawid et al. 2007; Kise et al. 2005; Carmer et al. 1996). However, only a few of them have been used to transform data streams from scanning LIDAR sensors into maps. Doerr (2009) and Doerr et al. (2014) reported a description and comparative evaluation of these methods (i.e. occupancy grid, raster scan, labeling, and average height). These studies concluded that the average height method was the most appropriate for minimizing the positioning error of obstacles present in various standing field crops.

Agricultural vehicles are subjected to random mechanical vibrations due to the machines' own mechanical systems, their operating speed, and field conditions (Balasubramanian 2006). Agricultural vehicles are generally designed without suspension systems; tires therefore represent the only suspension components working as both spring and damping elements (Clijmans et al. 1998). Many researchers have extensively studied vibrations on tractors using analytical and experimental approaches (Balasubramanian 2006; Dworecki et al. 2005; Clijmans et al. 1998) and have also observed the effect of vibration on LIDAR sensors (Lee and Ehsani 2008; Doerr 2009; Doerr et al. 2014). However, there remains a need to develop and evaluate appropriate stabilization systems that could reduce the effects of vibrations on LIDAR sensors.

The specific objectives of this study were: 1 . to design a mounting and stabilization system capable of reducing the effects of vibrations on a LIDAR sensor mounted on an agricultural tractor, and 2. to evaluate its effectiveness at improving the obstacle detection accuracy of the LIDAR sensor under different machine and field operating conditions.

\section{MATERIALS and METHODS}

A commercial Kubota B6200 agricultural tractor equipped with a LMS 291-S14 (SICK Ag, Germany) LIDAR sensor was used in this study.

LIDAR sensor, data acquisition, and stabilization system

The LIDAR sensor has an internal rotating mirror which reflects a pulsed laser beam to scan a path in front of the sensor (Figure 1). When this laser beam hits an object, part of the light is reflected back to a detector inside the scanner. The sensor measures the distance to objects by measuring flight time separating emitted and reflected laser pulse. The sensor allows for a fan-shaped two-dimensional scan of the surrounding area. The sensor has a $90^{\circ}$ sweep angle $\left(-45^{\circ}\right.$ to $+45^{\circ}$ relative to the direction of travel), an angular resolution of $0.5^{\circ}$, and a maximum range of $8.18 \mathrm{~m}$. The maximum range of the LIDAR unit was obtained by dividing the height of the LIDAR sensor on the tractor from the ground by the sine of the angle from the horizontal line $\left(8.9^{\circ}\right)$. Other significant characteristics of the sensor are: frequency or response time of $75 \mathrm{~Hz}(13.3$ $\mathrm{ms}$ ), resolution of $0.01 \mathrm{~m}$, and typical measurement accuracy of $0.035 \mathrm{~m}$ (Sensor Intelligence 2006).

Data output was via RS-422 Serial port and the serial data were converted to USB using the USB-COMi-M serial to USB converter. The LIDAR sensor data were logged on a laptop computer mounted on the tractor. Data were stored in a matrix file on the computer with each row of the matrix representing a separate scan, and each column of the matrix corresponding to each increment of sweep angle of the sensor. 


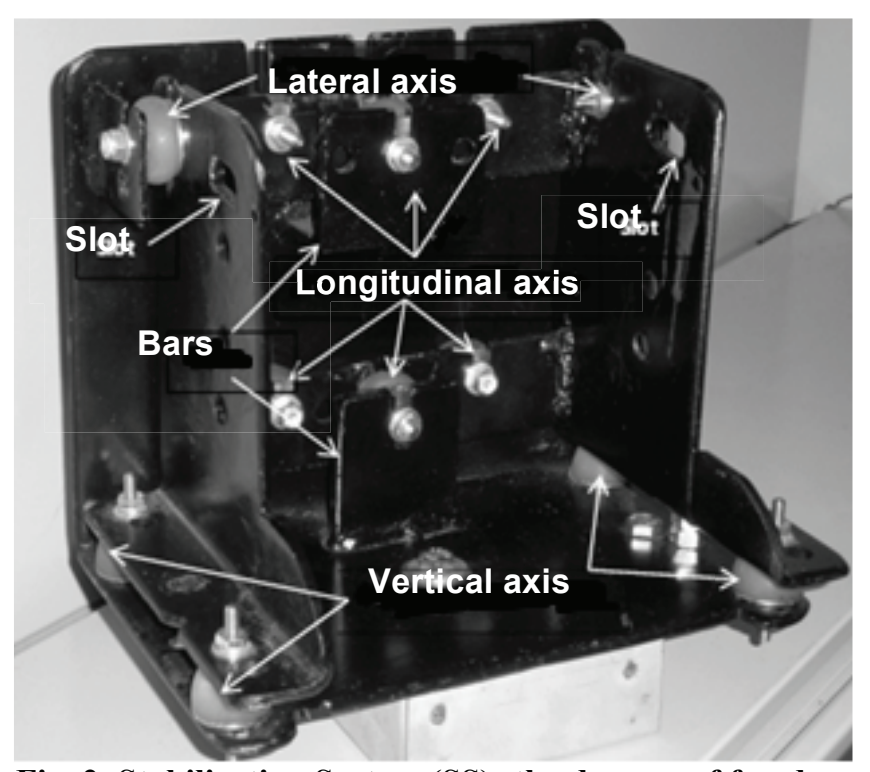

Fig. 2. Stabilization System (SS); the degrees of freedom addressed by the vibration isolators are indicated on the figure.

The LIDAR sensor was located above the hood and near the front of the tractor where it could have an unobstructed view of the path in front of and the sides of the tractor. However, this location is far away from the tractor centre of gravity, and is therefore subjected to both small amplitude high frequency and large amplitude low frequency vibrations resulting from translation (bounce) and rotation of the tractor about its centre of gravity.

A vibration isolation system was developed for the LIDAR sensor. Various types of vibration isolators were investigated and a particular type of isolator (V10Z64MN03 (Gelmec 2010, Norfolk, UK)) was chosen based on parameters such as frequency range, load capacity, geometric design and mounting position of the sensor, as well as environmental and material constraints. This particular isolator consists of an ultra-soft silicone gel that is bonded between two metal mounts. This gel has the following properties: $800 \%$ elongation, Young's Modulus equal to $156.2 \mathrm{kPa}$, and thermal conductivity of $0.80 \mathrm{Wm}^{-}$ ${ }^{1} \mathrm{~K}^{-1}$. Other important characteristics are: soft enough to damp to lower frequencies than rubber mounts, broad temperature range, extremely strong metal bond, and ideal for sensitive equipment.

The stabilization system (SS) incorporates 12 V10Z64-MN03 isolators installed along the three orthogonal axes (Figure 2). Six of them act along the longitudinal axis; four of them along the vertical axis; and the last two in the lateral direction. The isolators work in compression only, and pairs of opposing isolators were therefore used to absorb vibrations for each of the six degrees of freedom of the mounting system. Figure 3 illustrates the mounting of the LIDAR sensor on the stabilization system. Two parallel lateral plates were attached with two bars and bolted to the sensor. This

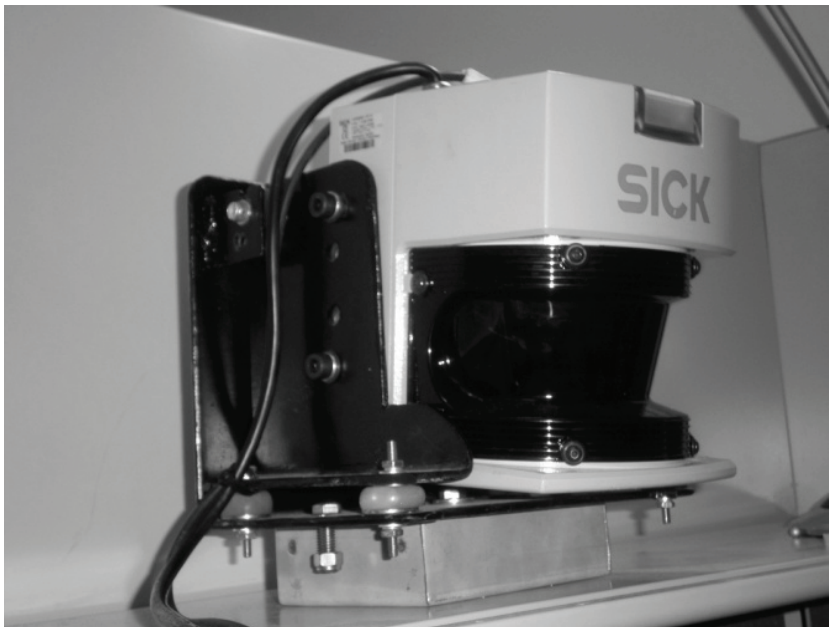

Fig. 3. SICK LMS 291-S14 scanner mounted on stabilization system.

assembly was attached to an angled base plate by the twelve isolators. The parallel lateral plates were designed so as to not obstruct the front viewing window of the sensor. The parallel lateral plates were provided with slots allowing the sensor to be angled downward by up to $15^{\circ}$.

A support structure was designed to support the LIDAR sensor on top of the tractor engine hood without adversely affecting operator visibility. The support structure was composed of two brackets bolted to the sides of the tractor chassis, two vertical bars bolted to the chassis brackets, and one horizontal bar extending over the hood and joining the vertical bars (Figure 4). Adequate clearance was provided between the components of the structure and the tractor pedals and steering linkage to avoid any interference. The mounting system allowed the LIDAR sensor to be moved in a vertical direction relative to the tractor chassis and centre of gravity. In order to further decrease longitudinal vibrations, two brackets were bolted to the front side of the tractor chassis and two support bars (S) were added to either side of the structure (Figure 4b).

\section{Experimental design, test course and protocol}

Field tests were carried out to evaluate the ability of the LIDAR system to detect obstacles for different configurations of the mounting systems and various fieldoperating conditions. The experimental site was located on the southeast corner of the Central Experimental Farm of Agriculture and Agri-Food Canada in Ottawa, Ontario.

Test tracks were set up in two different terrains classified according to surface roughness: smooth (grass-covered smooth field), and rough (recently harvested corn field), which were labelled 'ST', and 'RT' respectively (Figure 5). For the RT conditions, the tractor was driven perpendicular to the crop rows or furrows that acted as berms and generated large amplitude low frequency vibrations on the tractor. As shown on Figure 5, the RT berms had an average spacing of $0.75 \mathrm{~m}$ and average height of $0.05 \mathrm{~m}$. 

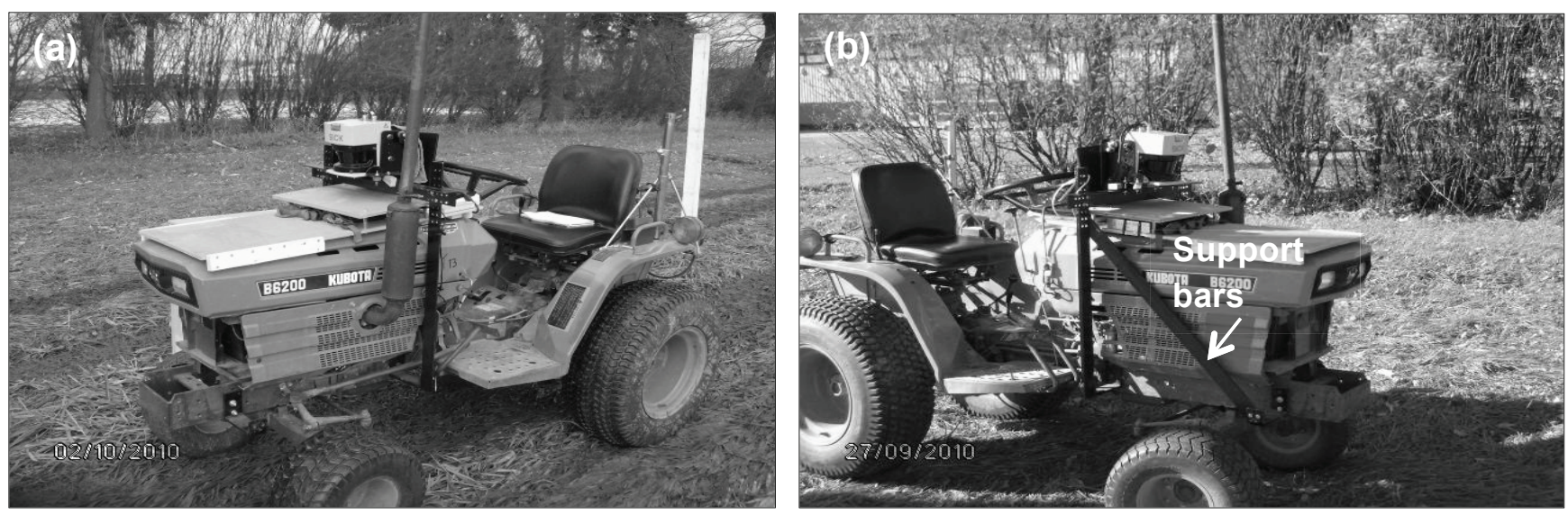

Fig. 4. LIDAR sensor structure. (a) without support bars, and (b) with support bars.

Four types of test objects made of different materials and having dissimilar shape, height, and colour were used as field obstacles. 1) a short square shaped box $(0.60 \mathrm{~m}$ wide by $0.78 \mathrm{~m}$ tall) labelled "box", 2) a tall square shaped post ( $0.09 \mathrm{~m}$ wide by $1.83 \mathrm{~m}$ tall) labelled "square", 3) a cylindrical object $(0.57 \mathrm{~m}$ diameter and $0.94 \mathrm{~m}$ tall $)$ labelled "cylinder", and 4) a tall rectangular post (cross section of $0.11 \mathrm{~m}$ by $0.04 \mathrm{~m}$ and height of $1.49 \mathrm{~m}$ ) labelled "rectangle". The material and colour of the objects were selected according to the recommendations on typical material reflectivity for this particular type of LIDAR sensor (Sensor Intelligence SICK 2006). The box and the rectangle were made from unpainted spruce plywood and unpainted spruce wood respectively but having the same coefficient of reflectivity of $40 \%$. The cylinder was made of PVC but it was wrapped with aluminum paper to increase its reflectivity to about $150 \%$. Surfaces that reflect the light as bundled (aluminium, steel, mirrored surfaces, reflectors) can be assigned a reflection value between 100 $2000 \%$ (Sensor Intelligence SICK 2006). The square was made of wood but it was painted in white and it had a reflectivity of $80 \%$. Figures $6 \mathrm{a}$ and $6 \mathrm{~b}$ show the four objects.

Table 1. Speed and support system combination.

\begin{tabular}{ccl}
\hline Treatment & $\begin{array}{c}\text { Speed } \\
(\mathrm{Km} / \mathrm{h})\end{array}$ & Support system \\
\hline 1 & 2 & S\&SS \\
2 & 2 & S\&nSS \\
3 & 2 & nS\&SS \\
4 & 2 & nS\&nSS \\
5 & 4 & S\&SS \\
6 & 4 & S\&nSS \\
7 & 4 & nS\&SS \\
8 & 4 & nS\&nSS \\
9 & 7 & S\&SS \\
10 & 7 & S\&nSS \\
11 & 7 & nS\&SS \\
12 & 7 & nS\&nSS \\
\hline
\end{tabular}

Here $\mathrm{n}$ refers to 'no' and S and SS refer to support bars and stabilization system respectively.
The field experiments were a factorial experimental design with three levels of operating speed $(2,4$ and 7 $\mathrm{km} / \mathrm{h}$ ), and four configurations of the LIDAR support system on the tractor (S\&SS, S\&nSS, nS\&SS and nS\&nSS, where $n$ refers to 'no' and S and SS refer to support bars and stabilization system respectively) (Table 1). Separate experiments of the same design were conducted on two types of terrain, smooth and rough. All experiments were repeated three times. A start flag was placed at the beginning of the test runs (i.e. $y=0 \mathrm{~m}$ ), followed by the box, the square, the cylinder, and the rectangle, which were placed at $4,8,12$, and $16 \mathrm{~m}$ respectively, and with the end flag placed at $20 \mathrm{~m}$. The four-meter distance between two objects or between an object and a flag was used to normalize the velocity error.

Figure 1 shows a plan view of the layout of the test runs with the obstacles placed to the side of the tractor path for detection and mapping by the LIDAR sensor. The tractor was driven at a constant speed past the start flag, the four objects and the end flag. Recording for each test began approximately $10 \mathrm{~m}$ before the start flag to allow the LIDAR sensor, which had a range $8.18 \mathrm{~m}$, to record the start flag from the bottom to the top, and recording was stopped a few meters after the tractor had passed the end flag. The length of the runs for each test was constant at 20 $\mathrm{m}$, although the total length of the map produced from the sensor data varied slightly among tests due to a small

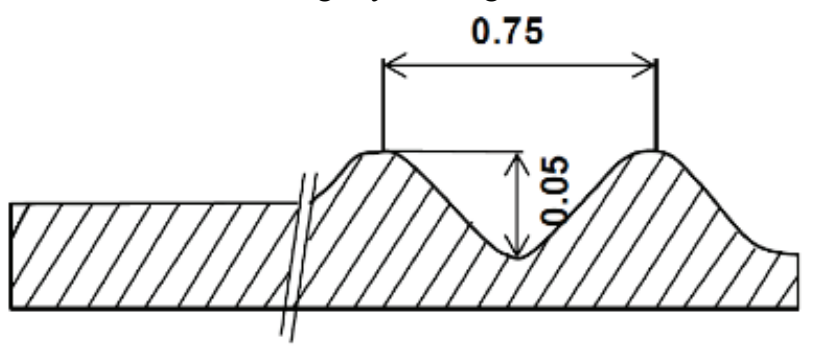

Smooth Terrain

Rough Terrain

\section{All dimensions are in $\mathrm{m}$}

Fig. 5. The dimensions of earthen berms for the two different terrains. 

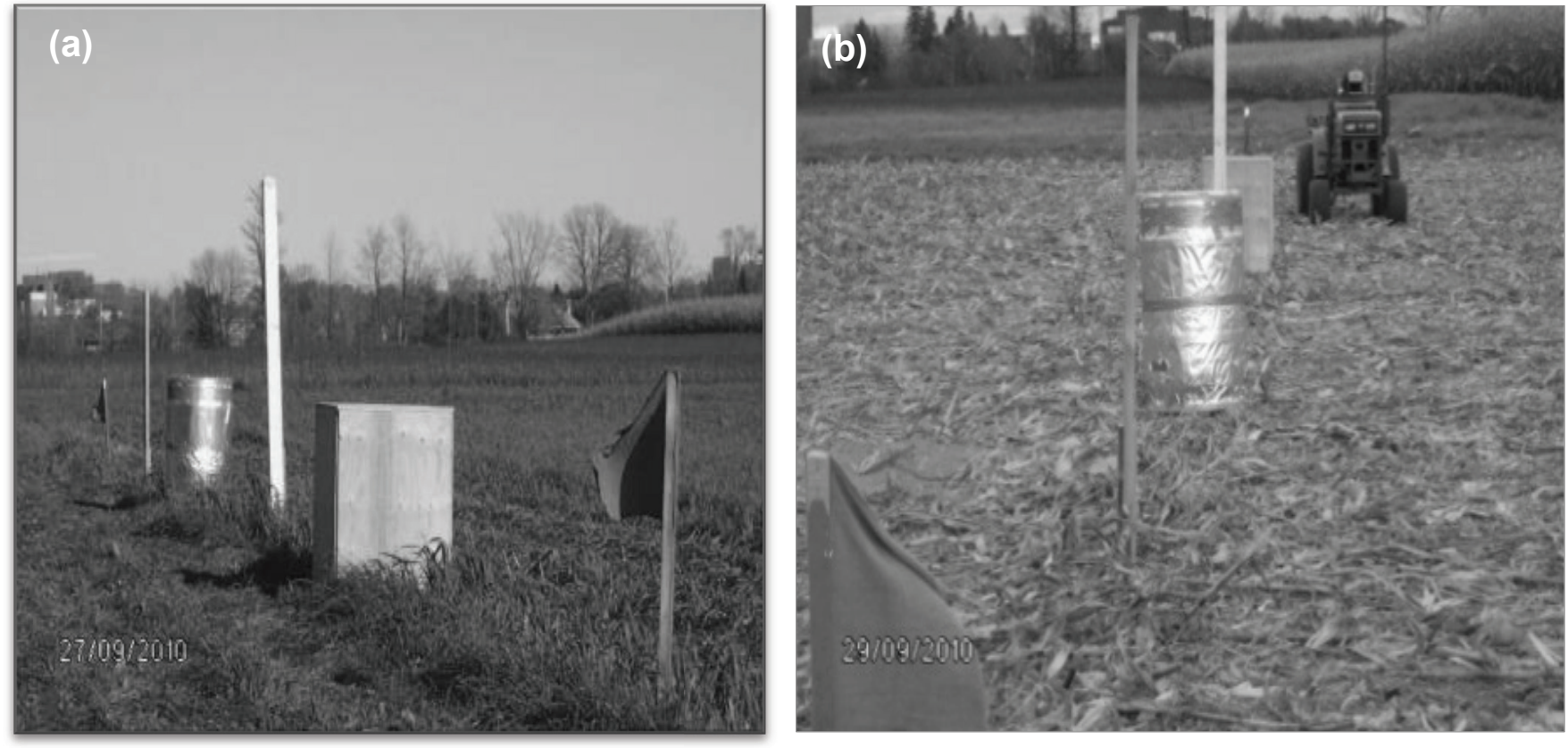

Fig. 6. The field tracks (a) the objects in smooth terrain, and (b) driving the tractor alongside the objects in rough terrain.

variability in tractor speed. The width of the maps created by the LIDAR sensor was $11.5 \mathrm{~m}$, corresponding to the scanning width, i.e. the distance between the points where the laser beam hit the soil surface at either end of the $\pm 45^{\circ}$ scan.
Data and image processing techniques

Figure 7 shows a simplified conceptual example for a series of 15 successive sensor-scans that would detect the four objects and the two reference flags. Each scan is represented by an inclined plane, and each column in the

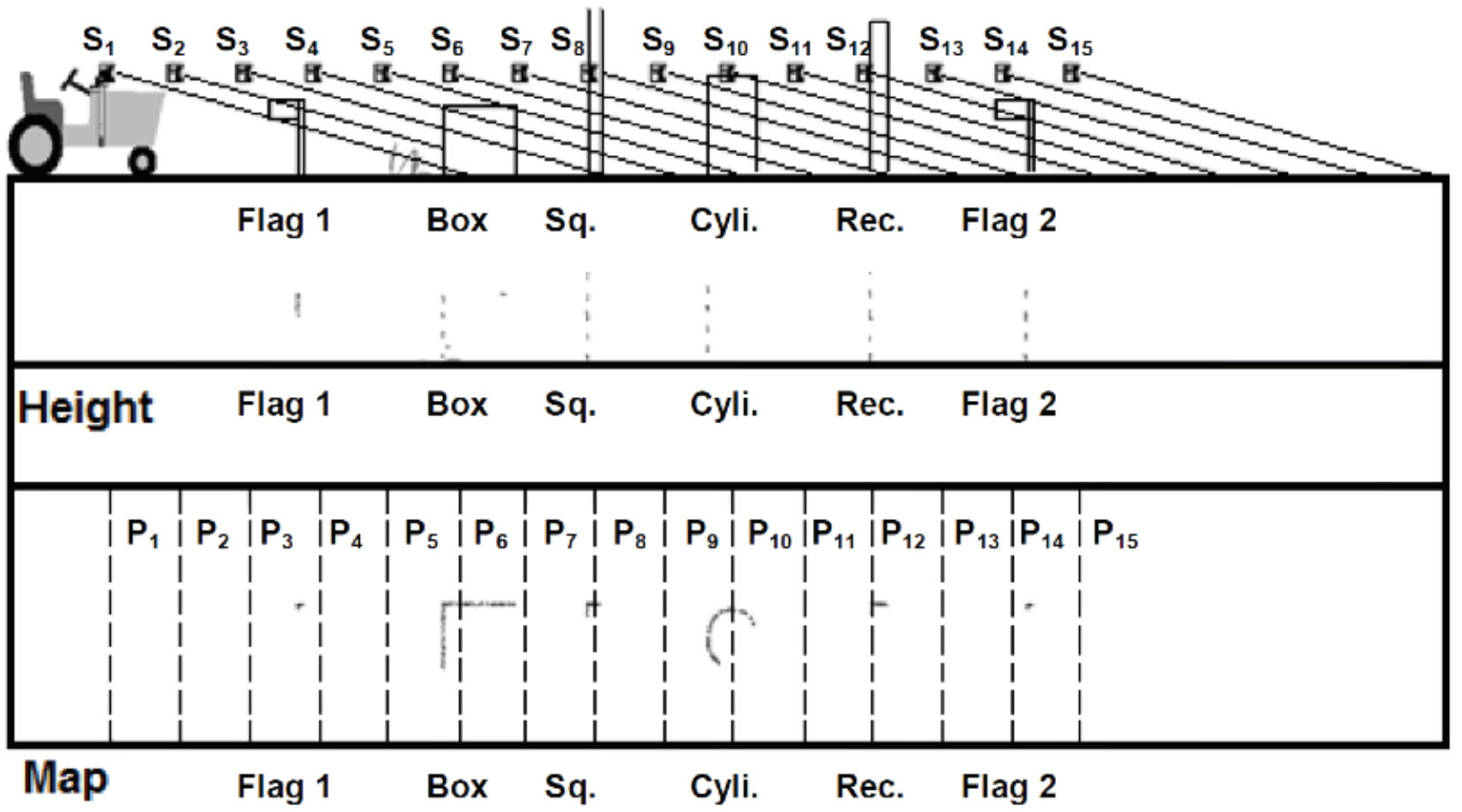

Fig. 7. Representation of 15 scans intersecting all of flag 1 (start), box, square, cylinder, rectangle and flag 2 (stop) and 15 corresponding planes that were subsequently used to create a map. 


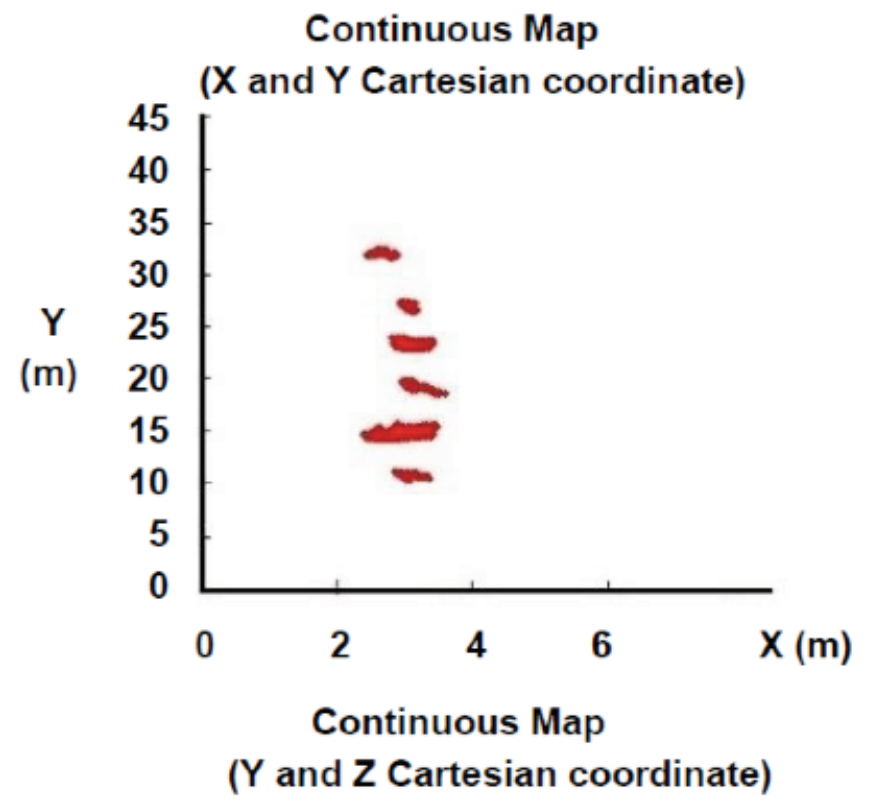

\author{
Image Map $X$ and $Y$ \\ (object center)
}

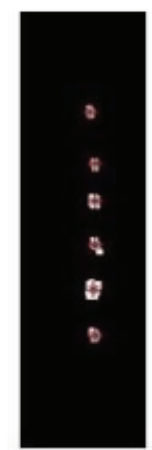

\title{
Image Map $Y$ and $Z$
}

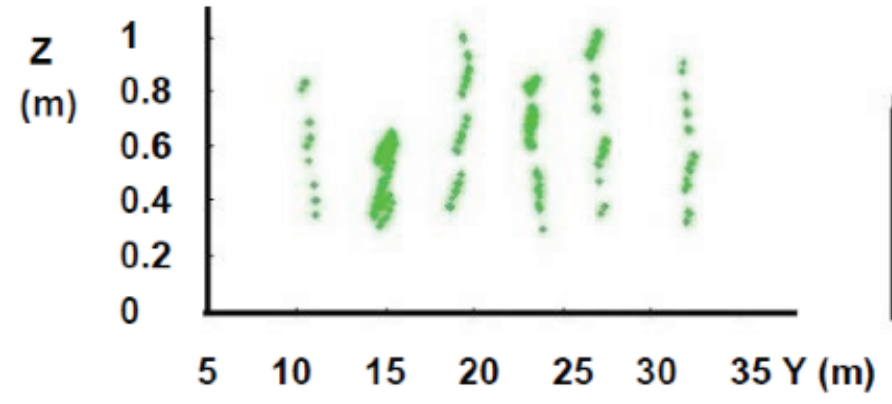

Fig. 8. Formation of images of the objects from the points (rough terrain test).

raw data matrix (different planes stacked parallel to the page, not shown) represents a different sweep angle of the sensor. Figure 7 illustrates how each object is detected at different vertical locations by several consecutive scans of the sensor. When an object is detected, the corresponding cell in the raw data matrix is said to be "occupied", with its value set as the scalar distance from the sensor to the object. The true object position in Cartesian coordinates relative to the start flag can therefore be determined from the scan number and tractor velocity, the sensor sweep angle, the scalar distance between the object and the sensor, and finally the geometry of the tractor and sensor mounting system.

The Cartesian coordinate system used in this study was as follows: $\mathrm{x}$-coordinate (lateral, to the right of the travel direction); $y$-coordinate (longitudinal, along the travel direction); z-coordinate (vertical). The start and end flags at either end of the test run were $20 \mathrm{~m}$ apart and provided reference points on the maps created by the LIDAR sensor. The true location of the objects on the maps was determined by interpolation between the images of the start and end flags on the maps. Sensing errors in the $\mathrm{x}$ and $\mathrm{y}$ directions were defined as the mean error distance between the respective actual and measured positions of the centroids of the objects. The object centroids were used as the object reference points, but the LIDAR detects the object front surface. Standard deviation of the measurement distance is a measure of variability or dispersion of data in a given data set about the mean, which was considered as a measure of inaccuracy or positioning error.

A Matlab program was developed to transform the raw data and create a continuous map of the field showing the location of the detected obstacles (Matlab 2011). The detected positions of the obstacles were obtained from the sensor data by means of the average height method (Doerr 2009). Using Matlab morphological functions, the images of the objects were later built by transforming the points represented in the continuous map into images. An image matrix was created for each plane and was represented with Xgrid and Ygrid values of $10 \mathrm{~cm}$, which corresponds to an image map pixel size of $10 \times 10 \mathrm{~cm}$. After that, the matrix was rotated and the Matlab functions "strel" was used to create morphological structuring elements according to each example. Then the functions dilate and erode were used to generate the images of the objects in the $x-y$ and $y-z$ planes. Similar methodology has also been used recently for semi-autonomous vehicle by Narayanaram et al. (2014). Figure 8 illustrates the formation of images of the objects obtained from the points in the continuous map in the $x-y$ and $y-z$ planes. 


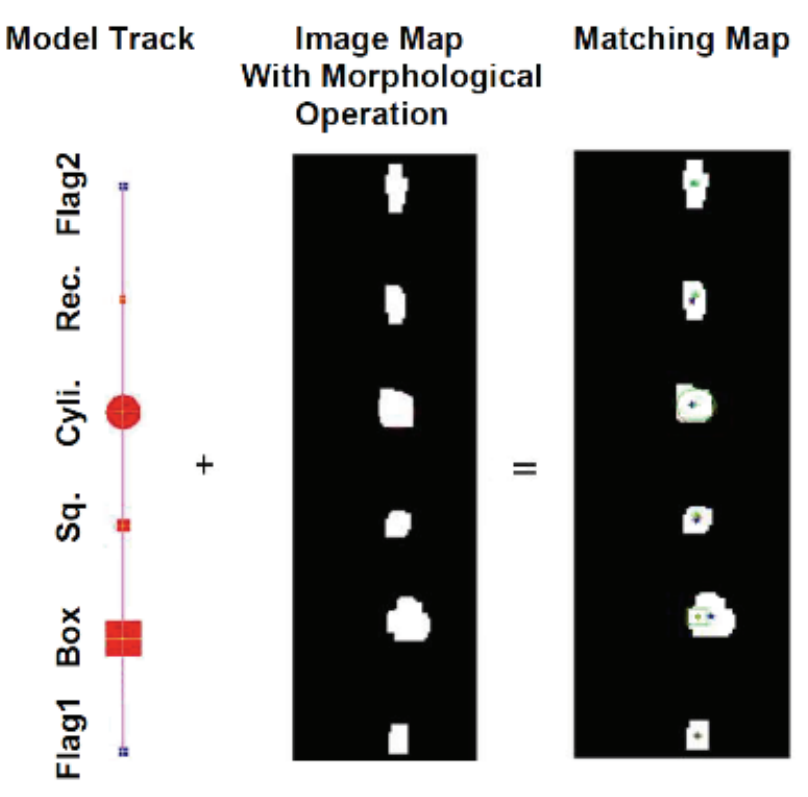

Fig. 9. Matching map with images of the objects in white and the real objects in + sign according the first approach and * sign according the second approach.

Data on the sensing error in the $\mathrm{x}$ and $\mathrm{y}$ directions (i.e. corresponding to variations in tractor roll and pitch respectively) were considered as dependent variables and subjected to analysis of variance using the General Linear Models procedure in SAS (SAS Institute 2012). The data were analyzed as a completely randomized factorial experimental design with three replicates. Means were considered to be significantly different at $\mathrm{p}<0.05$ according to the LSD test.

\section{RESULTS and DISCUSSION}

The experimental results were analyzed to determine the influence of the four independent variables (travel speed, type of terrain, support bars (S) and stabilization (SS) systems) on the obstacle positioning error of the LIDAR sensor for each obstacle. The accuracy of the sensor was deemed to be improved when both the average error distance and the standard deviations were reduced.

Figure 9 shows an example of a map matching of the model track with a map created by the LIDAR sensor. On this figure, the $\mathrm{x}$ and $*$ symbols represent the actual and measured centroids of the objects determined from interpolation between the reference flags, and measured by the sensor respectively. The two symbols were forced to superimpose for the start and end flags (bottom and top images respectively on Figure 9) by the software. For the four other obstacles, the "*" and "+" symbols did not exactly match, therefore indicating the differences between the actual and measured positions of the obstacles. The scalar distance between these two positions corresponds to the measurement error.

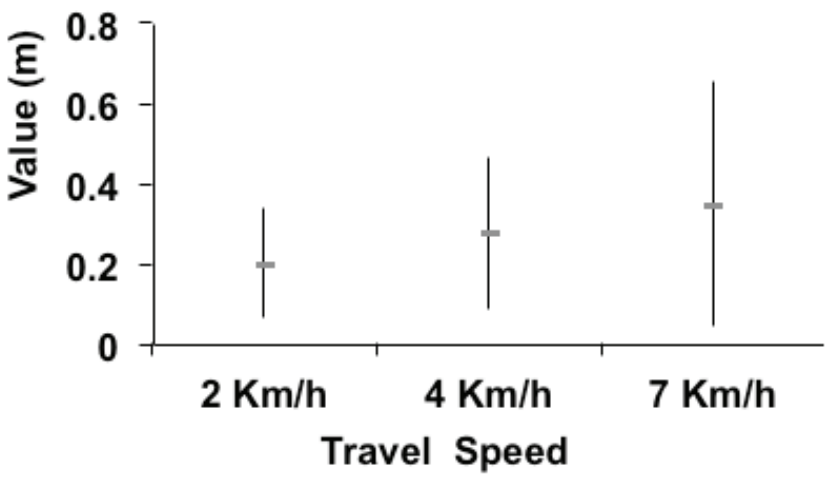

Fig. 10. Mean error distance (scalar value) and standard deviation at different tractor travel speeds.

\section{Effects of Travel Speed}

The average positioning error and standard deviations increased with increasing travel speeds (Figure 10). The mean error increased from $20 \mathrm{~cm}$ at $2 \mathrm{~km} / \mathrm{h}$ to $35 \mathrm{~cm}$ at 7 $\mathrm{km} / \mathrm{h}$, while the standard deviation increased from $14 \mathrm{~cm}$ at $2 \mathrm{~km} / \mathrm{h}$ to $30 \mathrm{~cm}$ at $7 \mathrm{~km} / \mathrm{h}$. This is in agreement with the work of Lee and Ehsani (2008), who reported more noise in laser measurements when vehicles were traveling at high speed.

Statistical analyses were also completed for the obstacle positioning error in the $\mathrm{x}$ and $\mathrm{y}$ directions (i.e. perpendicular and parallel to the direction of travel respectively). In the case of the longitudinal positioning error, significant differences were observed for the square and rectangular objects $(\mathrm{p}<0.05)$, for the box object $(\mathrm{p}<$ $0.01)$, and for the cylinder test object $(\mathrm{p}<0.001)$ (data not shown). Conversely, the lateral positioning error was not significantly affected by operating speed. These results are consistent with those obtained by Abdullah and AL-Mafraji (2011) who found that as the travel speed was increased from 2 to $6 \mathrm{~km} / \mathrm{h}$, the vertical, longitudinal, and lateral accelerations at the location of the tractor seat also increased. They found that the increased longitudinal acceleration was at least twice the vertical and lateral acceleration. Higher operating speed results in increased longitudinal acceleration and therefore higher variations of the distance between the LIDAR sensor and the objects.

\section{Effect of Terrain}

The mean error distance and standard deviation were analyzed under both Smooth Terrain (ST) and Rough Terrain (RT) operating conditions. The average error decreased with increasing terrain roughness slightly from $29 \mathrm{~cm}$ for ST to $27 \mathrm{~cm}$ for RT, while the standard deviation increased from $16 \mathrm{~cm}$ to $28 \mathrm{~cm}$. Figure 11(a) (ST) and 11(b) (RT) show the results (mean error distance and standard deviation) for the different configurations of the sensor mounting system. Particularly, the lowest values of mean error distance were observed with both the support bars (S) and stabilization system (SS) in place while larger values of obstacle positioning errors were obtained without 

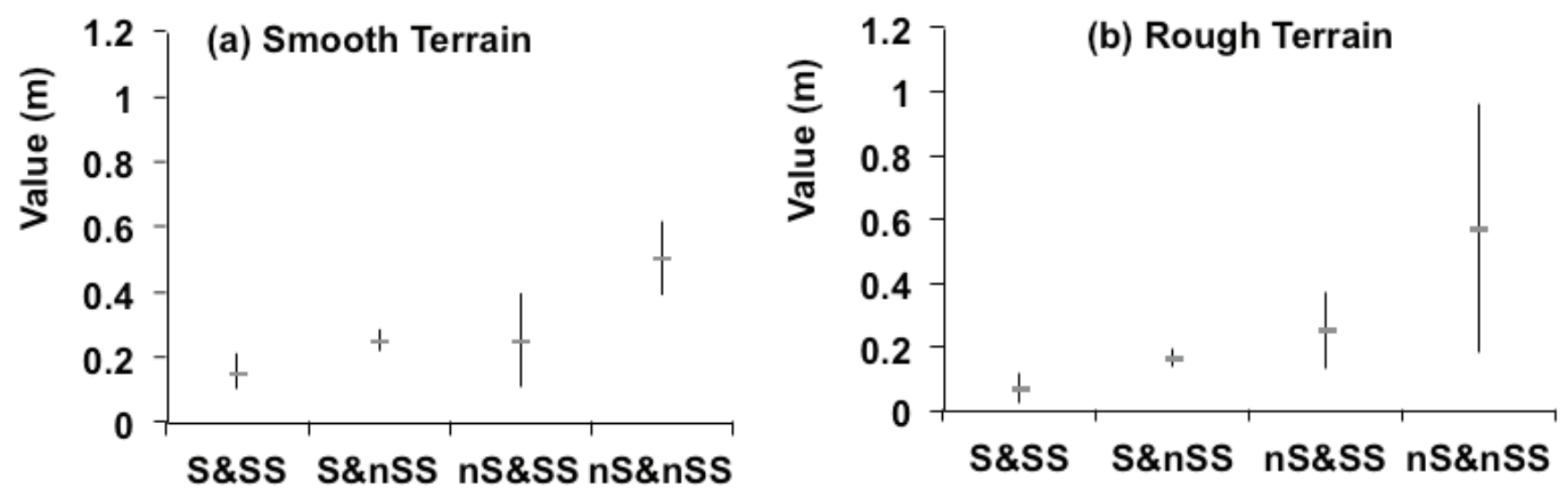

Fig. 11. Mean error distance (scalar value) and standard deviation.

the S and SS systems. Under both surface conditions, the addition of the stabilization system (SS) reduced mean error distance values between 36 to $56 \%$ compared to the results observed without the stabilization system (nSS). Similar results were obtained with tests performed with and without the support bars ( $\mathrm{S}$ and $\mathrm{nS}$ ) with a 38 to $71 \%$ reduction in the mean error distances when the support bars were present.

For all four types of test objects, the $\mathrm{x}$ error (perpendicular to the direction of travel) was much larger for smooth terrain than for rough terrain while the error in the direction of travel (y) was similar for both terrains. Much of the measurement errors in the $\mathrm{x}$ and $\mathrm{y}$ direction were likely due to variations in tractor roll and pitch respectively which would result in corresponding variations of the distance from the LIDAR sensor to the object. These results show that tractor roll had more effect on the sensor performance than tractor pitch. This is also in agreement with the work of Dworecki et al. (2005) who investigated the interior tractor vibration and found the valuated error of pitch moment and roll moment of tractor inertia were 4.8 and $9.8 \%$, respectively. Kumar et al. (2001) measured the vertical, longitudinal and lateral tractor seat vibrations under varying terrain conditions (road surface, village path and farm). They found that the longitudinal vibrations were $46 \%$ less than the lateral on a road surface, $21 \%$ on a village path and $13 \%$ on a farm terrain.

\section{Effect of Support Bars (S)}

Figure 12(a) shows the overall effects of the support bars on the values of mean error distance and standard deviation. The mean error distance increased from $20 \mathrm{~cm}$ with the support bars in place to $34 \mathrm{~cm}$ when they were removed, which represents a $40.9 \%$ increase (standard deviation: from 16 to $25 \mathrm{~cm}$ or $+37.3 \%$ ). These results demonstrate that the LIDAR sensor was more accurate at detecting the location of the objects with the presence of the support bars than without them. This was attributed to the support bars reducing the vibration levels and resulting motion of the sensor with respect to the tractor chassis.

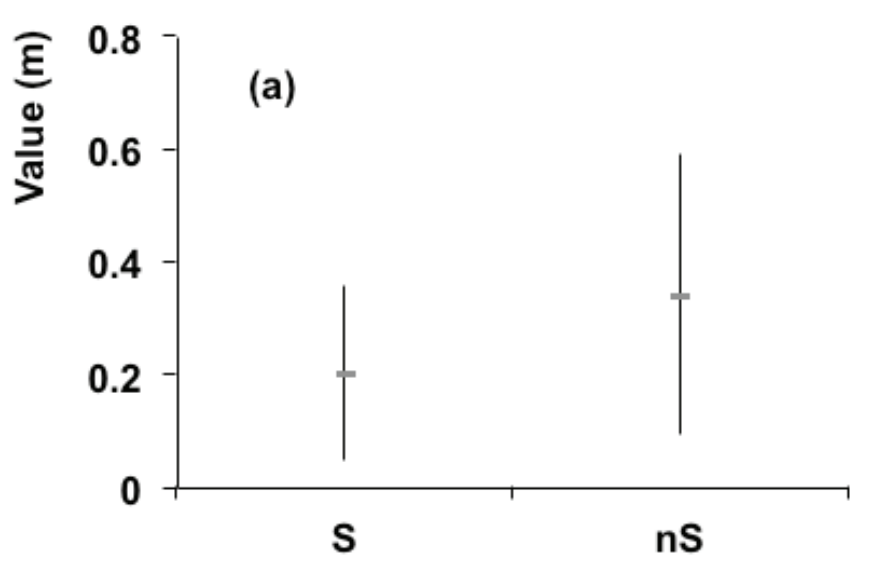

Support Bars

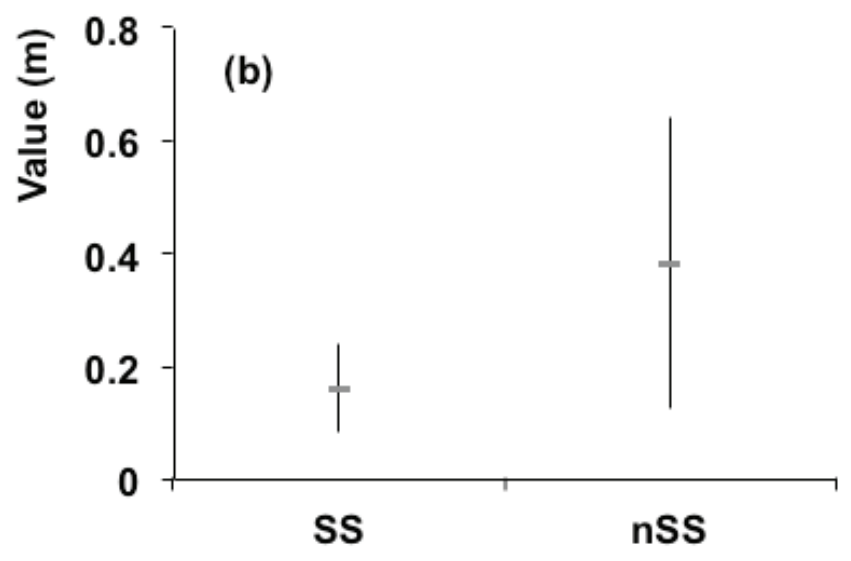

Stabilization System

Fig. 11. Mean error distance (scalar value) and standard deviation a) with and without support bars (S), b) with and without SS. 


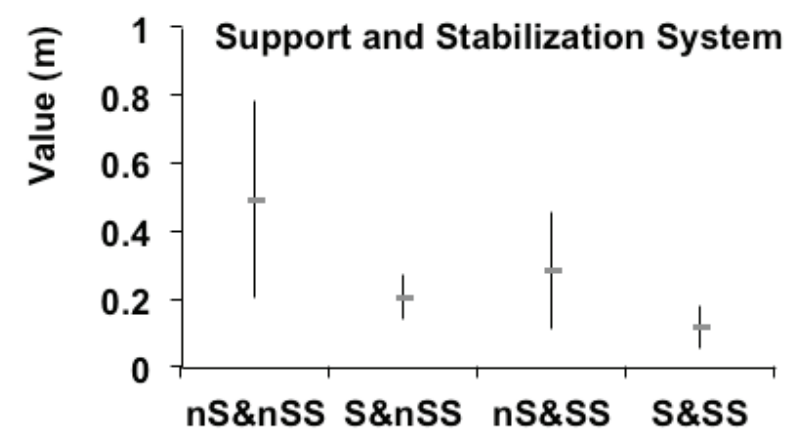

Fig. 13. Mean error distance (scalar value) and standard deviation with and without support bars (S) and stabilization system (SS).

Therefore, the support bars reduced measurement noise and positioning error introduced by vibrations.

\section{Effects of Stabilization System (SS)}

Figure 12(b) shows the mean error distance and standard deviation values observed with and without the stabilization system. Both the mean error distance and standard deviation values increased significantly when the stabilization system was not present; the mean error distance increased from 17 to $38 \mathrm{~cm}$ and the standard deviation increased from 8 to $26 \mathrm{~cm}$. These represent relative increases of 56.6 and $69.5 \%$ respectively. These results indicate that the use of the stabilization system can decrease the positioning error of the LIDAR sensor.

Combined effects of the Support Bars (S) and of the Stabilization System (SS)

As indicated on Figure 13, the best results in terms of low error distance and standard deviation were obtained when the LIDAR mounting system incorporated both the support bars and stabilization system. These two components allowed for reduced measurement noise caused by vibrations.

\section{CONCLUSIONS}

1. This study demonstrated that an increase in tractor speed resulted in an increase in both the mean error distance between the actual and detected location of the obstacles and the standard deviation. The addition of support bars (S) to the mounting system of the LIDAR sensor allowed for an increase in the accuracy of the sensor with both the mean error distance decreasing by $41 \%$ from 34 to $20 \mathrm{~cm}$, and the standard deviation decreasing by $37 \%$ from 25 to $16 \mathrm{~cm}$. Similarly, the incorporation of the stabilization system (SS) to the mounting system of the LIDAR also improved its accuracy with the mean error distance decreasing by $57 \%$ from 38 to $17 \mathrm{~cm}$ and the standard deviation values decreasing by $69 \%$ from 26 to $8 \mathrm{~cm}$.

2. This study confirms the effectiveness of incorporating both the stabilization system (SS) and support bars (S) in improving the accuracy of the LIDAR sensor mounted on an agricultural tractor under different field operating conditions through a reduction in transmitted vibrations by 40 to $60 \%$. Therefore, this type of design could increase the accuracy and safety of autonomous agricultural vehicles for essential tasks such as guidance and obstacle detection.

\section{ACKNOWLEDGEMENT}

The authors thank the Natural Sciences and Engineering Research Council of Canada for financial support of this research and the Eastern Cereal and Oilseed Research Centre of Agriculture and Agri-Food Canada for the use of their resources and facilities.

\section{REFERENCES}

Abdullah, M.K. and A.A.H. AL-Mafraji (2011). Measurement of transmitted vibration to tractor seat. Journal of Engineering 5 (17):1260-1268.

Balasubramanian, K., T.F. Burks., C.J. Lehtola and W.S. Lee. 2006. Shock and vibration data acquisition system for off-road vehicle operator health and safety assessment. Journal of Agricultural Safety and Health 12(4): 293-313.

http://dx.doi.org/10.13031/2013.22010

Barawid Jr, O.C., A. Mizushima., K. Ishii and N. Noguchi. 2007. Development of an autonomous navigation system using a two-dimensional laser scanner in an orchard application. Biosystems Engineering 96 (2): 139-149. http://dx.doi.org/10.1016/j.biosystemseng.2006.10.01 2

Burdick, H.E. 1997. Digital Image, Theory and Applications, New York, NY. McGraw-Hill, Inc.

Carmer, D.C. and L.M. Peterson. 1996. Laser radar in robotics. Proceedings of the IEEE 84 (2): 299-320. http://dx.doi.org/10.1109/5.482232

Clijmans, L., H. Ramon and J. De Baerdemaeker. 1998. Structural modification effects on the dynamic behaviour of an agricultural tractor. Transactions of the ASABE 41(1): 5-10.

http://dx.doi.org/10.13031/2013.17145

Doerr, Z. 2009. Evaluating the ability to detect foreign objects in crops using laser range scanners mounted on agricultural vehicles. Unpublished M.A.Sc. Thesis. Department of mechanical engineering, faculty of engineering, University of Ottawa, Ottawa, ON.

Doerr, Z., A. Mohsenimanesh., C. Laguë and N.B. McLaughlin. 2013. Application of the LIDAR technology for obstacle detection during the operation of agricultural vehicles. Canadian Biosystems Engineering 55: 2.9-2.16. http://dx.doi.org/10.7451/CBE.2013.55.2.9

Dworecki, Z., A. Fiszer., M. Loboda and J. Przybyl. 2005. Calculation of tractor and agricultural machines moments of inertia. Journal of Research and Applications in Agricultural Engineering 50(3): 2023. 
Fresquet Periu, C. 2012. Isolating vibrations on laser range scanners mounted on agricultural vehicles to improve the detection of foreign objects. Unpublished M.A.Sc. Thesis. Department of Mechanical Engineering, faculty of engineering, University of Ottawa, Ottawa, ON.

Gelmec. 2010. Gelmec vibration, shock, noise and thermal control solutions. Gelmec.co.uk. http://www.gelmec.co.uk (2010/02/15).

Kise, M., Q. Zhang and N. Noguchi. 2005. An obstacle identification algorithm for a laser range finder based obstacle detector. Transactions of the ASAE 48(3): 1269-1278. http://dx.doi.org/10.13031/2013.18491

Kumar, A., P. Mahajan., D. Mohan and M. Varghese. 2001. Tractor vibration severity and driver health. Journal of Agricultural Engineering Research 80 (4): 313-328. http://dx.doi.org/10.1006/jaer.2001.0755

Lee, K.H. and R. Ehsani. 2008. Comparison of two 2D laser scanners for sensing object distances, shapes, and surface patterns. Computers and Electronics in Agriculture 60 (2): 250-62.

http://dx.doi.org/10.1016/j.compag.2007.08.007
Li, M., K. Imou., K. Wakabayashi and S. Yokoyama. 2009. Review of research on agricultural vehicle autonomous guidance. International Journal of Agricultural \& Biological Engineering 2 (3).

Matlab. 2011. User guide. Mathworks.com. http://www.mathworks.com (2011/03/12).

Narayanaram, A., A. Sridhar and K.N. Prakash. 2014. Simulation of navigation system for semiautonomous vehicle. IOSR Journal of Electrical and Electronics Engineering e-ISSN: 2278-1676, pISSN: 2320-3331: 6-8.

Sensor Intelligence, SICK. 2006. LMS200/211/221/291 Laser measurement systems (The technical description for the MST200 Measurement Software Tool). Waldkirch: SICK.

Subramanian, V., T.F. Burks and A.A. Arroyo. 2006. Development of machine vision and laser radar based autonomous vehicle guidance systems for citrus grove navigation. Computers and Electronics in Agriculture 53(2): 130-143.

http://dx.doi.org/10.1016/j.compag.2006.06.001 